\section{Contemporary orthodontics: the micro-screw}

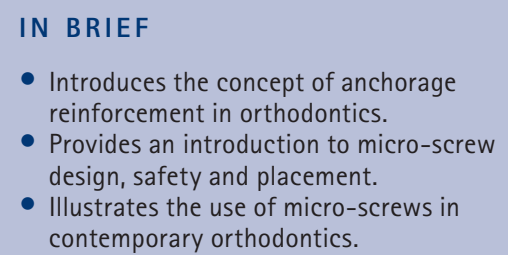

M. 0. Sharif*1 and D. T. Waring ${ }^{2}$ contemporary orthodontics.
VERIFIABLE CPD PAPER

Micro-screws (MSs) have gained rapid popularity among orthodontic specialists over the past decade. Subsequently, as general dental practitioners (GDPs) continue to provide general care for patients undergoing orthodontic treatment they are likely to encounter MSs. This article is aimed at GDPs and provides an overview of MS design, placement, removal and safety. Two examples of treated cases will also be used to demonstrate the use of MSs in contemporary orthodontic practice.

\section{INTRODUCTION}

The concept of using implants in orthodontics has existed for over 50 years. ${ }^{1}$ The micro-screw (MS) is a type of dental implant; they are also commonly referred to as miniimplants or temporary anchorage devices (TADs). The first clinical use of 'screws' in orthodontics was by Creekmore and Eklund in $1983,{ }^{2}$ who reported on the insertion of a vitallium bone screw into the anterior nasal spine to treat a patient with a deep overbite. The advent (in the late 1990s) of microscrews that are relatively easy to place and well tolerated ${ }^{3}$ has led to their rapid gain in popularity among orthodontic specialists over the past decade. In contrast to conventional dental implants the contemporary MS relies on mechanical retention and not osseointegration for stability and retention and invariably it can be loaded immediately. They are temporarily fixed within bone and are commonly used to provide 'anchorage' that is, resistance to unwanted tooth movement. ${ }^{4}$ As every action has an equal and opposite reaction (Newton's third law) the concept of anchorage is of particular importance in orthodontics, because if a force is applied to a tooth/a group of teeth then an equal and opposite force will also be generated. This opposite force commonly results

School of Dentistry, University of Manchester, Manchester, M13 9PL; ${ }^{2}$ University Dental Hospital of Manchester, Higher Cambridge Street, Manchester, $\mathrm{M} 156 \mathrm{FH}$

*Correspondence to: Mohammad Owaise Sharif Email:mohammad.sharif@manchester.ac.uk

\section{Refereed Paper}

Accepted 12 March 2013

DOI: 10.1038/sj.bdj.2013.376

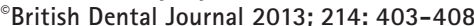

in tooth movement. In cases where this reactionary movement is unwanted anchorage needs to be 'reinforced'. Common methods for achieving this are to use:

- Differential segments of teeth

- Extraction of teeth

- Bone (for example a trans-palatal arch)

- Headgear

- Functional appliances.

None of the above methods result in complete resistance to unwanted tooth movement. Because micro-screws are fixed directly within bone they theoretically provide absolute anchorage and therefore eliminate all unwanted tooth movement. Their success rate has been reported to be in the region of $80 \% .^{5}$

- Micro-screws are commonly used to aid in the following:

- Retraction of anterior teeth

- Anterior open bite correction

- Intrusion of teeth

- Extrusion of teeth

- Alignment of impacted teeth

- Up-righting of tipped teeth

- Creation of space for restorative treatment in hypodontia cases.
This article aims to provide an overview of the general MS design, placement, removal and safety. Two examples of treated cases will be used to demonstrate the use of micro-screws in contemporary orthodontic practice.

\section{MICRO-SCREW DESIGN}

Micro-screws are generally manufactured from a titanium alloy or stainless steel ${ }^{6}$ and each generally has three distinct sections:

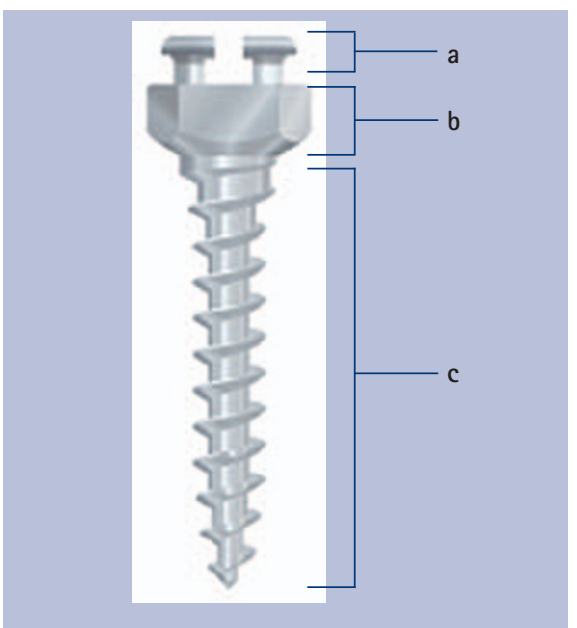

Fig. 1 Sections of a MS: (a) head, (b) collar, (c) thread

\begin{tabular}{|l|l|l|l|}
\multicolumn{3}{|c}{ Table 1 A comparison of the features of three commonly used MS systems } \\
\hline & Infinitas (db Orthodontics) & Vector (Ormco) & Abso Anchor (Dentos) \\
\hline Material & Titanium 6-4 & Titanium 6-4 & Titanium 6-4 \\
\hline Head design & Cross head with undercuts & Double delta with eyelet & Various head designs \\
\hline Thread type & Self-drilling & Self-drilling & Self-drilling \\
\hline Body length $(\mathrm{mm})$ & $6-9$ & $6-12$ & $5-12$ \\
\hline Collar width $(\mathrm{mm})$ & $1-2$ & 2 & $1.2-2.7$ \\
\hline MS diameter $(\mathrm{mm})$ & $1.5-2$ & $1.5-2$ & $1.2-2.7$ \\
\hline
\end{tabular}


- The head (supra-gingival, allows for attachment)

- The collar (trans-mucosal)

- Thread (inta-osseous, provides mechanical retention).

Figure 1 demonstrates the various sections of a MS.

Three MSs commonly used in the UK are the 'Infinitas Mini Implant System' (db Orthodontics), the 'Vector Temporary Anchorage System' (Ormco) and the 'Abso Anchor' system (Dentos). The main features of these three systems are demonstrated in Table 1.

The main difference between the various MS systems available is head design. It is reported that the Infinitas cross-head is versatile and allows attachment of 'stock' closing coil springs and elastomeric chains. ${ }^{7}$ The Vector double-delta head MS on the other hand is reported to provide increased comfort, in addition the availability of MS specific attachments with swiveling heads is said to reduce 'torsional and rotational forces. ${ }^{8}$ The differences in head design can be readily identifiable as demonstrated in Figure 2.

Microscrews are also available in various lengths (6-12 mm); the length selected varies depending on site and method of insertion. In general a short MS (6 $\mathrm{mm}$ ) would be selected for perpendicular insertion in the anterior maxilla region whereas a longer MS (9 or $12 \mathrm{~mm}$ ) would be used for insertion in the palate or infrazygomatic crest.

Micro-screws may be self-tapping or selfthreading. Self-tapping MSs require a pilot hole to be made before insertion whereas the self-threading variety do not. One technique for the placement of a self-threading MS is discussed in the next section.

\section{MICRO-SCREW PLACEMENT AND REMOVAL}

A detailed knowledge of anatomy is a pre-requisite to the placement of an MS. Micro-screws are inserted close to the muco-ginigval margin but within the zone of attached gingivae (that is, into keratinised tissue). If the MS is placed further occlusally its stability is compromised (as the volume of bone is reduced), conversely placing the MS further towards the sulcus will increase the volume of bone available. However, alveolar mucosa is friable and is prone to tearing.

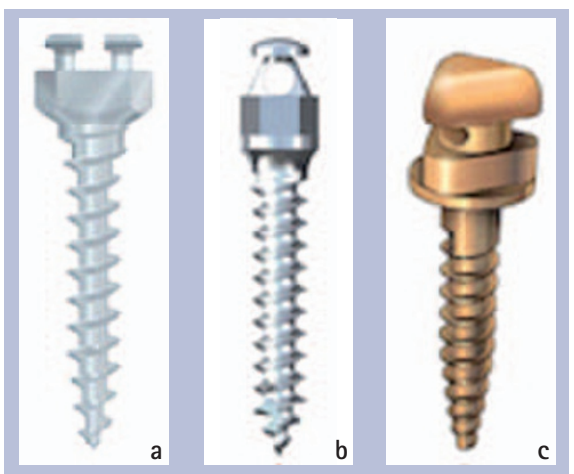

Fig. 2 Three commonly used MS systems: (a) Infinitas, (b) Abso Anchor, (c) Vector. The differences in head design are readily identifiable

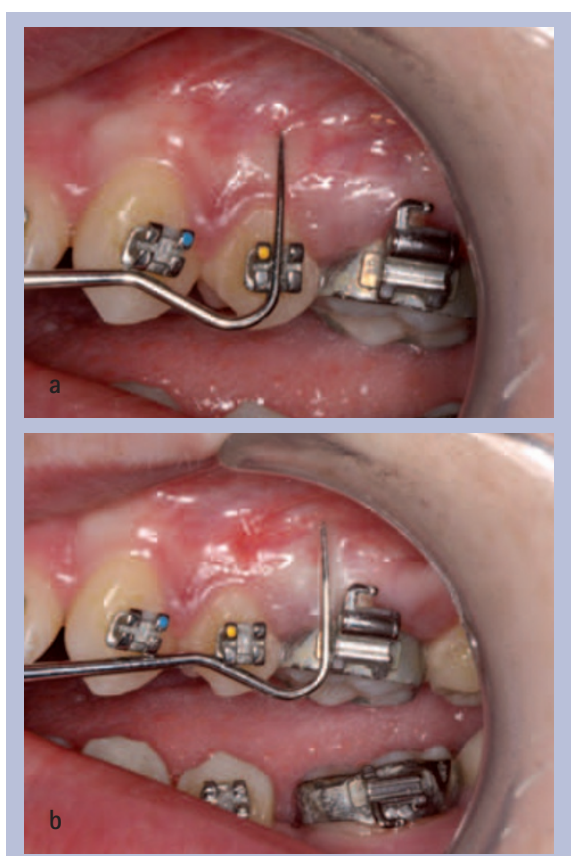

Figs 3 and 4 The long axes of the 25 and 26 teeth are located using the centre of the 25 bracket and the mesial aspect of the 26 tube. NB: This was done after assessment of the preoperative OPT to confirm the absence of abnormal anatomy/ obstructions in the 25-26 region (the desired site for MS placement in this case)

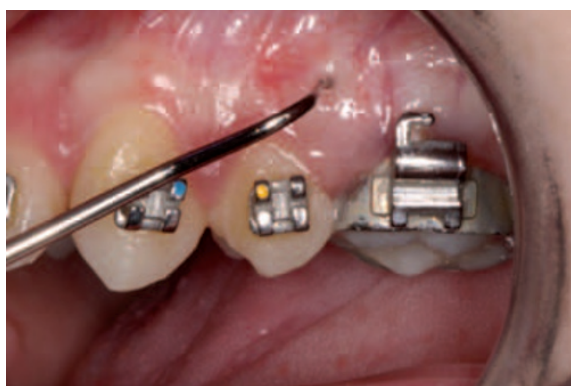

Fig. 5 The use of a probe to demarcate the MS placement site

In addition, placement of the screw may be more difficult due to 'loose' moveable mucosa that can become entangled in the MS body as it is inserted.

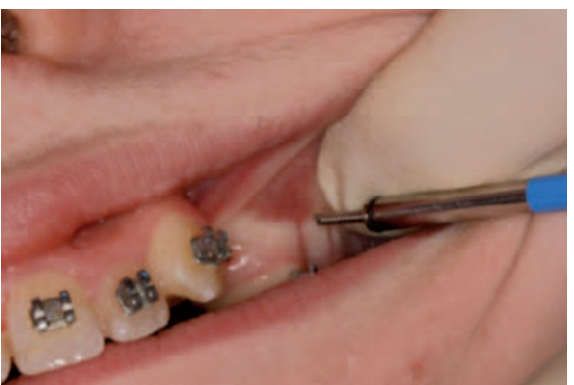

Fig. 6 Placement of the MS using a manufacturer provided driver

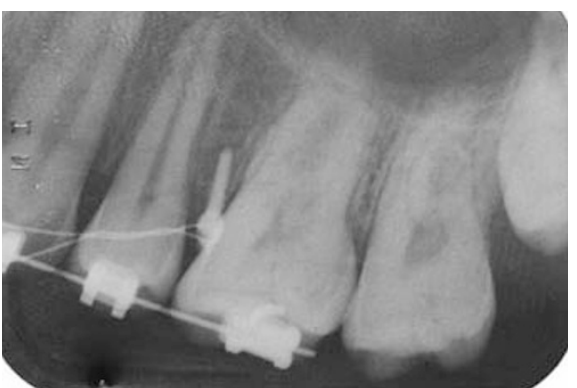

Fig. 7 A check PA radiograph demonstrating the MS in situ, clear of adjacent roots and within bone

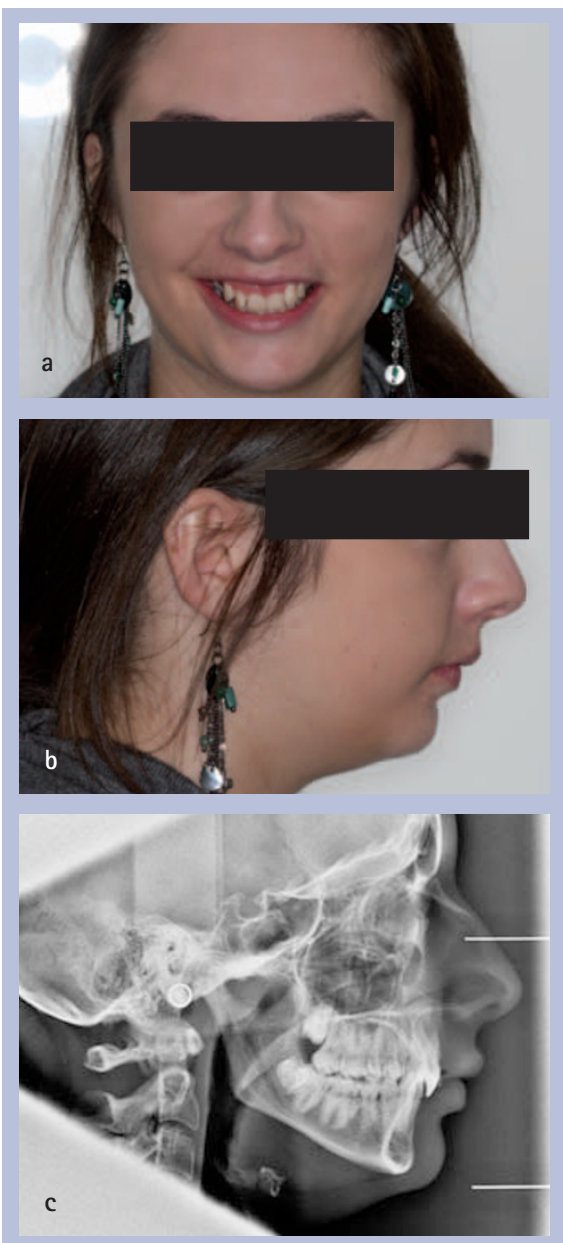

Fig. 8 (a) Extra-oral view - smiling; (b) extra-oral view - profile; (c) lateral cephalometric radiograph. This supports the clinical findings of retroclined upper incisors, Class II skeletal patter and a reduces FMPA) 


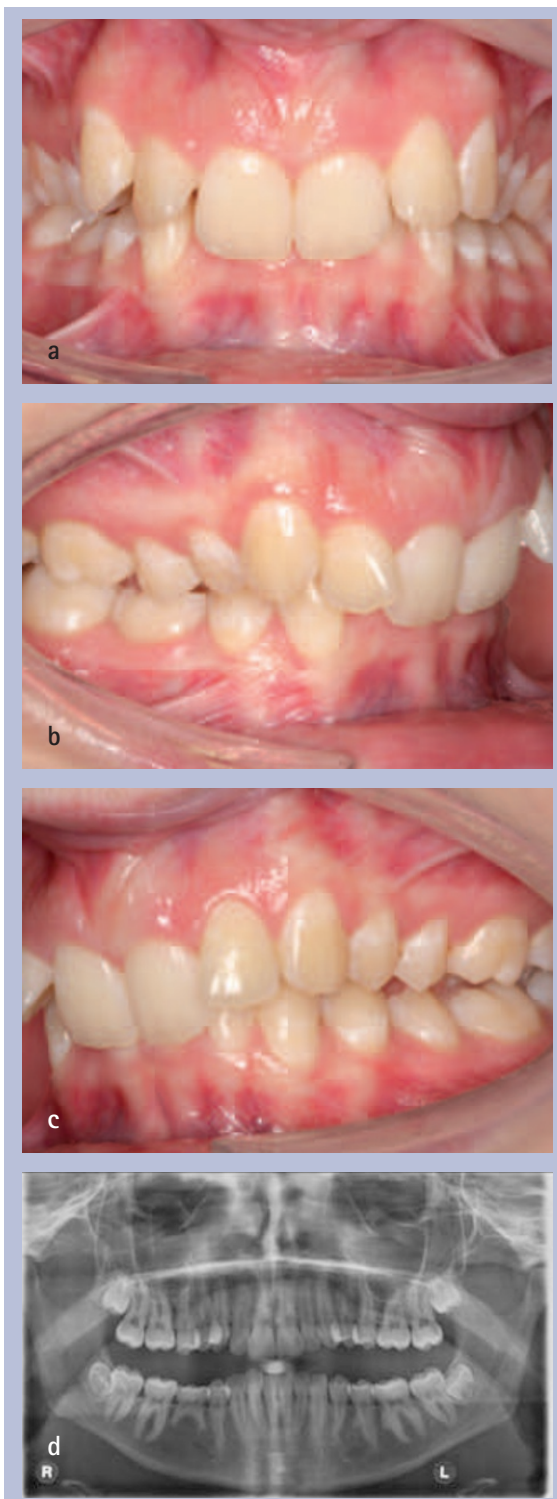

Fig. 9 (a-c) Pre-treatment intra-oral views (front occlusal, right buccal and left buccal respectively); (d) OPG - showing good bone levels, good root length and morphology and the presence of all permanent teeth except the $\mathbf{4 5}$

When considering a site for MS placement it is important to assess the presence of abnormal anatomy and obstructions (for example converging roots). Orthopantomograms (OPTs) commonly inform orthodontic treatment planning and are therefore readily available to screen for obstructions and abnormal anatomy.

Before the placement of a MS the patient is advised to gargle with a chlorhexidinebased mouthwash for one minute. Effective local anaesthesia is needed for MS placement; typically a topical anaesthetic agent is applied using a cotton wool roll for two minutes before the administration of a buccal infiltration to obtain anaesthesia. Half a

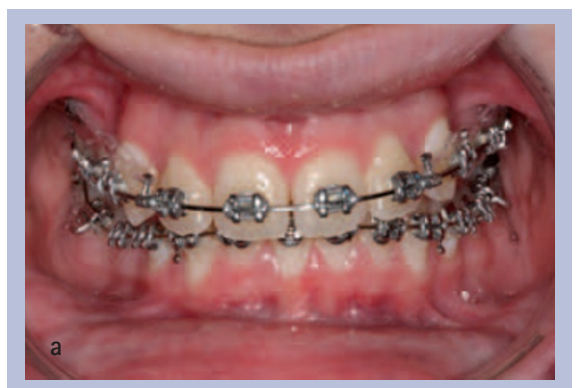

complications are as follows:

- Root contact and/damage on insertion

- MS fracture

- Pain

- Infection

- Nerve damage.

The National Institute for Health and Clinical Excellence (NICE) has published guidance on the use of MSs for orthodontic anchorage, ${ }^{6}$ this highlighted the lack of major safety concerns.

\section{CONSENT}

Informed consent is a prerequisite for any clinical procedure and MS insertion is no exception. As alluded to earlier in the text, MSs are not always successful and successful dental alignment cannot be guaranteed since several other interrelated factors are associated with this, for example, patient compliance and regular attendance.

For consent to be valid, appropriate information must be provided to the patient about their proposed procedure, its risks and benefits and also the alternative treatments available. ${ }^{9}$ The anchorage demands of a case are determined at the treatment planning stage (generally several months before insertion) and therefore patients are advised of the requirement of MS use (if appropriate) at this point. In our clinical settings it is standard protocol to discuss the risks, benefits and alternative options at this stage and provide patients with the British Orthodontic Society's patient information leaflet titled Orthodontic mini-screws. ${ }^{10}$ Consent is reconfirmed before MS insertion.

\section{CASE EXAMPLES}

\section{Case one}

A protocol for placing a MS is demonstrated in Figures 3-7.

Occasionally towards the end of their use MSs can become loose, this does not preclude their use and makes removal a very simple procedure. Frequently, all that is needed is topical anaesthetic, this allows for the MS to be removed by screwing it counter-clockwise. For firm MSs the anaesthetic technique described for MS insertion is utilised and the screw subsequently removed by screwing it counter-clockwise.

\section{SAFETY}

Complications associated with MSs are extremely rare, ${ }^{6}$ however, potential
An 18-year-old female was referred by her GDP to the orthodontic department. Her main complaint was crowding associated with both the upper and lower arches. On examination extra-orally she presented with a Class II skeletal pattern with reduced vertical proportions (Figs 8a-c). Intra-orally she presented with a Class II division 2 incisor relationship, crowding in both upper and lower arches, an increased overbite and a developmentally absent 45 (Figs 9a-d). In addition there was a reduction of lower tooth show on smiling and excess maxillary gingivae show (Fig. 8a). The patient was 
unconcerned about her Class II skeletal profile and requested treatment to correct the malocclusion only. This patient was deemed to be beyond the age at which functional appliances could be utilised.

The treatment plan utilised was:

1. Upper removable appliance to reduce the overbite and allow lower fixed appliance placement

2. Upper and lower fixed appliances

3. Extraction of upper first pre-molars

4. Aim to create Class I incisor relationship

5. Aim for Class II molar relationship (maintaining the 45).

\section{Treatment progression}

To aid treatment and reinforce anchorage, a MS was placed in both the upper right and upper left quadrants. The MSs were loaded immediately; the attached power chain was joined from the MS to ball hooks on the upper $19 \times 25$ " stainless steel archwire, this was to obtain overjet reduction (Figs 10a-c).

In this case all the space from the extracted upper first pre-molars was needed to permit overjet reduction and obtain a Class I incisor relationship. The MSs were invaluable as they allowed retraction of the upper labial segment (upper 3-3) without the loss of anchorage that is, without forward movement of the upper molar teeth. At the end of treatment, the profile appeared to have slightly improved although this is likely to be due to further growth rather than the effect of the fixed orthodontic treatment (Figs 11a and b). Overall, an excellent occlusion was obtained and upper and lower fixed and removable retainers were fitted to maintain the alignment (Figs 12a-e).

\section{Case two}

A 23-year-old male medical student was referred by his GDP for a joint orthodontic/ restorative opinion. His main complaint was the poor aesthetic appearance of his upper anterior teeth. Extra-orally he presented with a Class I skeletal pattern and acceptable tooth show on smiling, however, it was evident that only one central incisor (21) was visible (Fig. 13). Intraorally, the 11 was displaced and partially erupted, there was no history of trauma, it was therefore concluded that the $11 \mathrm{had}$ never developed in the correct position and
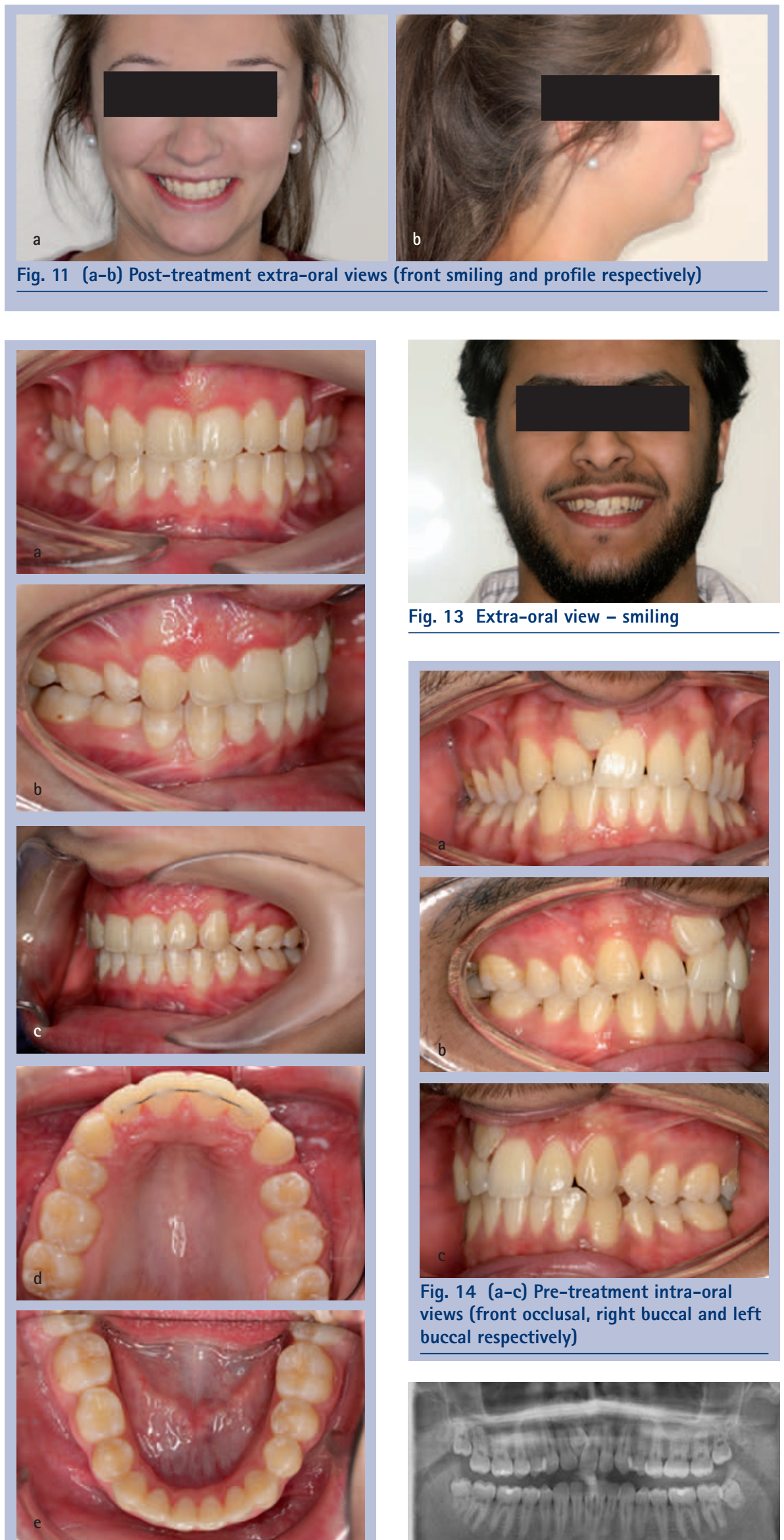

Fig. 12 (a-e) Post-treatment intra-oral views (front occlusal, right buccal and left buccal, maxillary and mandibular views respectively)
Fig. 13 Extra-oral view - smiling
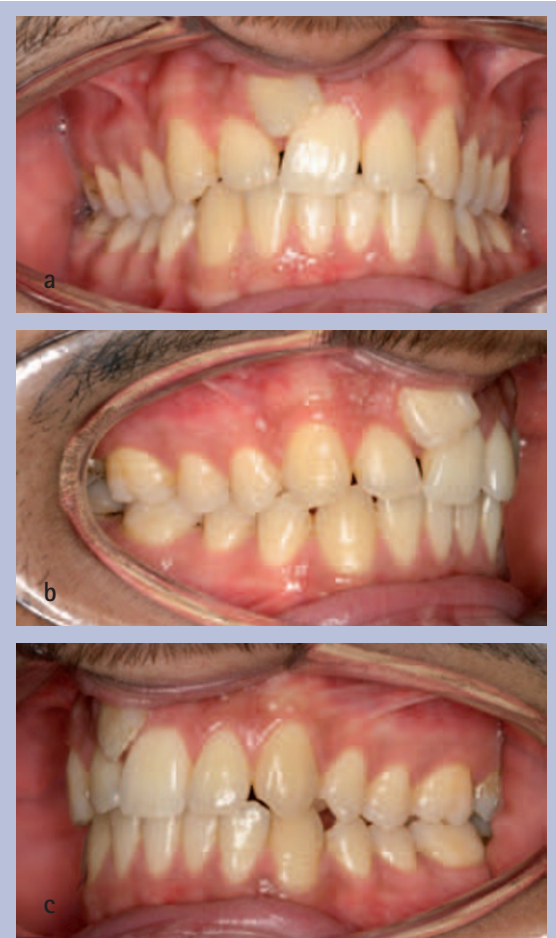

Fig. $14(\mathrm{a}-\mathrm{c})$ Pre-treatment intra-oral views (front occlusal, right buccal and left buccal respectively)

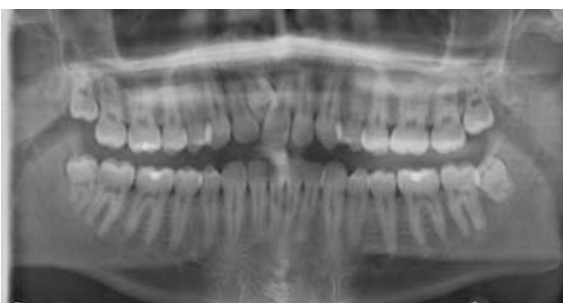

Fig. 15 OPG - showing good bone levels, good root length and morphology and the presence of all permanent teeth 
so the region of the 11 was taken up by the 12 and 21. The patients' incisor relationship was Class I, his right buccal segment was a Class I and the left buccal segment was a Class II (Figs 14a-c). The upper centre line had significantly deviated to the right. Radiographic examination revealed an impacted 11 with a suggestion of reduced root length (Fig. 15). The presence of perio-dontal ligament space around the 11 suggested that the tooth was unlikely to be ankylosed.

A treatment plan was discussed to attempt alignment of the 11 . There was an obvious need to create space to accommodate the displaced 11 and correct the deviated centre-line.

The treatment plan utilised was:

1. Upper and lower fixed appliances to create space and align the 11

2. Extraction of upper left first pre-molar

3. Aim to create Class I incisor/s

4. Aim for Class II molar/s on the left side

5. Maintain the Class I molar/s on the right side

6. Correct the centre line.

\section{Treatment progression}

All of the space created by extracting the 24 was needed to correct the centre line and create space in the 11 region. A MS was therefore utilised to aid correction, this meant that space could be opened and was not lost as the molar segments remained undisturbed (Figs 16a and b). The MS allowed retraction of the 13 to create space for the 11. Once sufficient space was created the 11 was engaged in the appliance system and alignment obtained (Figs 17a and b). Once the 11 was in the correct position and the space closure completed the appliances were removed and retainers fitted. A good outcome was achieved (Figs 18a-f) and a fixed retainer was bonded to the central incisors.

It is important to note that if 11 was deemed to be of unfavourable prognosis (ankylosed, dilacerated etc) the treatment plan detailed could have been used to create space for the placement of an implant, removable partial denture or bridge.

\section{CONCLUSION}

The micro-screw is a significant advancement in orthodontics. Micro-screws can be used for a variety of indications and
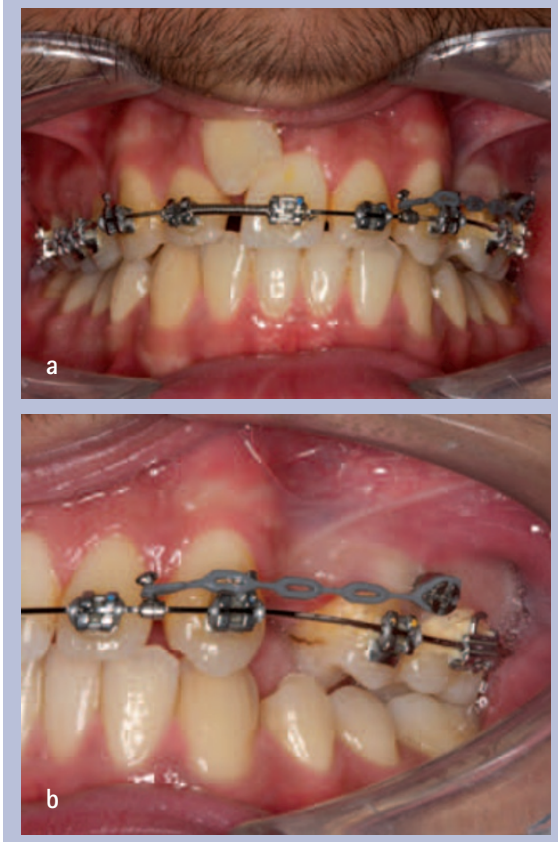

Fig. $16(\mathrm{a}-\mathrm{b})$ In-treatment intra-oral views (front occlusal and left buccal respectively - showing pushcoil in 11 region to open space and power chain on the left side from the MS to a hook on the upper archwire)
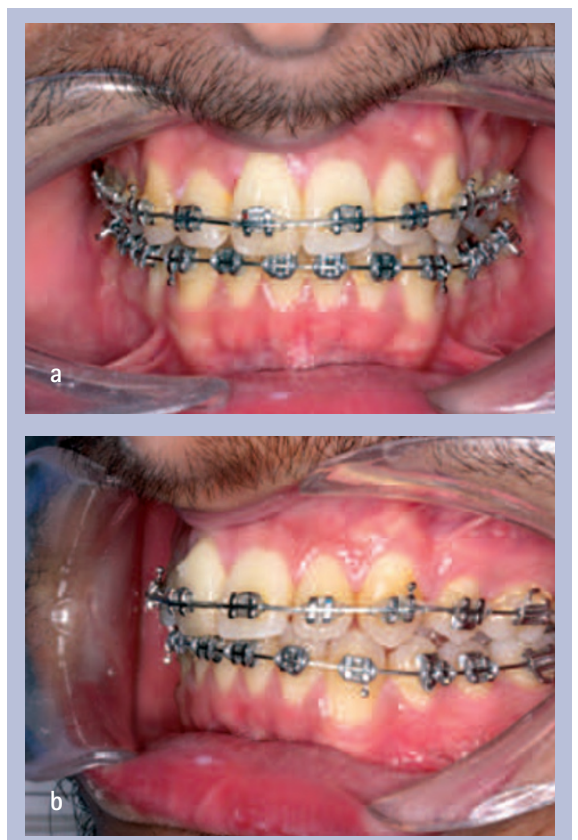

Fig. 17 (a-b) In-treatment intra-oral views (front occlusal, left buccal views respectively)

importantly in some instances act as a non-compliance device when compared with alternatives (for example, headgear). Although evidence related to effectiveness is limited at present, recent research has yielded promising results ${ }^{11-13}$ and therefore it is conceivable that the GDP will encounter microscrews in a growing number of patients.
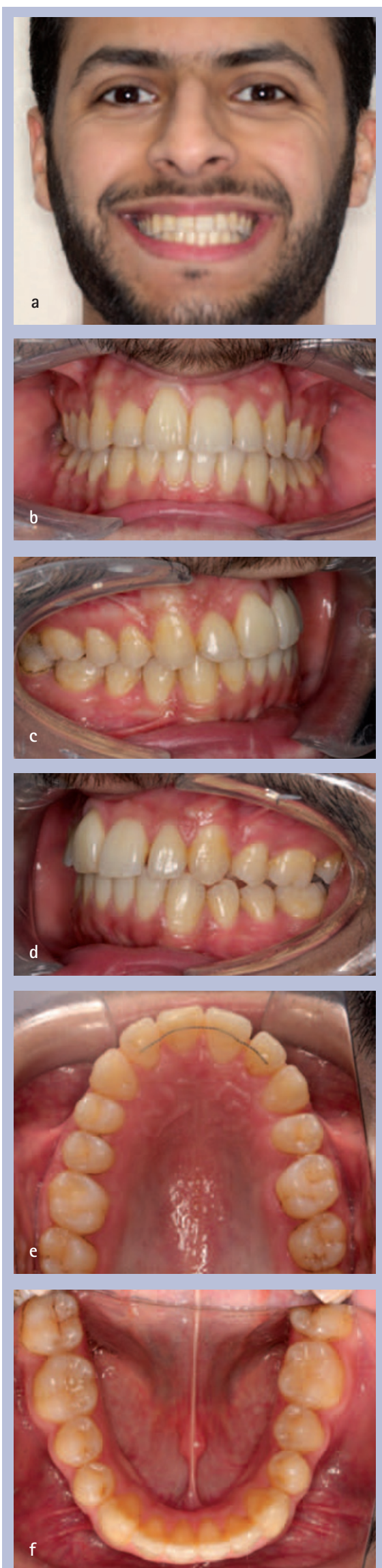

Fig. 18 (a) Post-treatment extra-oral view (front smiling); (b-f) post-treatment intraoral views (front occlusal, right buccal and left buccal, maxillary and mandibular views respectively) 
The authors would like to thank Susi Caldwell (consultant orthodontist) and Richard Needham (StR orthodontics), Manchester, for kindly providing the images utilised in Figures 3-7. The author are also grateful to $\mathrm{db}$ Orthodontics, Ormco and Torque Orthodontics for providing images for use in Figures 1 and 2 as well as confirming the information informing Table 1.

1. Gainsforth B L, Highley L B. A study of orthodontic anchorage possibilities in basal bone. Am J Orthod Oral Surg 1945; 31 : 406-417.

2 Creekmore T D, Eklund M K The possibility of skeletal anchorage. J Clin Orthod 1983; 17: 266-269.

3. Cousley R R J. Changing the face of orthodontics with mini-implants. Faculty Dent J 2012; 3: 34-41.

4. Daskalogiannakis J. Glossary of orthodontic terms: Leipzig: Quintessence Publishing, 2000.

5. Moon C H, Park H K, Nam J S, Im J S, Baek S H.
Relationship between vertical skeletal pattern and success rate of orthodontic mini-implants. Am J Orthod Dentofac Orthop 2010; 138: 51-57.

6. National Institute for Health and Clinical Excellence. Mini/micro screw implantation for orthodontic anchorage. London: NICE: 2007. Online article available at http://www.nice.org.uk/IPG238 (accessed March 2013).

7. Infinitas mini-implant system. Product guide. West Yorkshire: DB Orthodontics Ltd, 2010. Online guide available at http://www.infinitas-miniimplant. co.uk/pdfs/infinitas-sales-brochure.pdf (accessed March 2013).

8. Ormco. VectorTAS Temporary Anchorage System CA: Ormco, 2012. Online information available at http://www.ormco.com/products/vectortas/simplesystem.php (accessed March 2013)

9. Department of Health. Reference guide to consent for examination or treatment, London: DH, 2001.
Online article available at http://www.health.wa.gov. au/mhareview/resources/documents/UK_reference quide.pdf (accessed March 2013).

10. British Orthodontic Society. Orthodontic miniscrews: patient information leaflet. BOS, 2012.

11. Upadhyay M, Yadav S, Nagaraj K, Patil S. Treatment effects of mini-implants for en-masse retraction of anterior teeth in bialveolar dental protrusion patients: a randomized controlled trial. Am J Orthod Dentofac Orthop 2008; 134: 18-29.

12. Feldmann I, Bondemark L. Anchorage capacity of osseointegrated and conventional anchorage systems: a randomized controlled trial. Am J Orthod Dentofac Orthop 2008; 133: 339.

13. Cornelis M A, Scheffler N R, De Clerck H J, Tullock $J$ F, Behets C N. Systematic review of the experimental use of temporary skeletal anchorage devices in orthodontics. Am J Orthod Dentofac Orthop 2007; 131: S52-S58.

\section{Erratum}

Obituary (BDJ 2013; 214: 321)

\section{'Muriel E. H. Davis'}

In the above obituary, the author's name should have read as follows: Julia E. C. Davis

We apologise for any misunderstanding caused. 